\title{
Safety and effectiveness of intravitreal injection of dexamethasone for macular edema secondary to retinal vein occlusion: a meta-analysis
}

\section{Gao Xiaorong}

Affiliated Hospital of Southwest Medical University

\section{Dan Yujiao}

Affilited Hospital of Southwest Medical University

Chen Jie

Affiliated Hospital of Southwest Medical University

\section{Tian Gang}

Affilited Hospital of Southwest Medical University

Yue He ( $\nabla$ hylyfy@163.com )

Affiliated Hospital of Southwest Medical University

\section{Research article}

Keywords: Dexamethasone implant, meta-analysis, Ozurdex, retinal vein occlusion secondary macular edema (RVO-ME)

Posted Date: January 17th, 2020

DOI: https://doi.org/10.21203/rs.2.21159/v1

License: (c) (1) This work is licensed under a Creative Commons Attribution 4.0 International License. Read Full License 


\section{Abstract}

Background: This meta-analysis aimed to investigate the efficacy and safety of intravitreal injection of dexamethasone (DEX) in treating macular edema secondary to retinal vein occlusion.

Method: PubMed, Embase-clinical key, clinicaltrials.gov, Web of Science, and SinoMed were searched to compare the application of DEX implant in patients with retinal vein occlusion secondary macular edema (RVO-ME). The central retinal thickness, best-corrected visual acuity (BCVA), postoperative intraocular pressure, and postoperative cataract were extracted. RevMan 5.3 was used to analyze and evaluate the data.

Results: This study was based on six randomized trials. A total of 393 eyes were studied. DEX implant could effectively reduce macular edema caused by retinal vein occlusion. After 6 months, the drug could significantly improve the patients' BCVA [95\% confidence interval (Cl), 9.669-21.649, P = 21.649]. At the same time, it also could significantly reduce the patients' central retinal thickness (95\% Cl: $274.965-$ $142.236, P=0.0000)$. DEX implant could increase the intraocular pressure in some patients and greatly increase the risk of cataracts.

Conclusions: DEX implant can effectively improve the BCVA of patients with RVO-ME, reduce the central thickness of the retina, and reduce the number of injections for a relatively long time. Therefore, intravitreal injection of DEX can be used as an effective method for treating RVO-ME.

\section{Background}

Retinal vein occlusion (RVO) is one of the common vascular diseases of the fundus, which is also a common cause of blindness. The main manifestation of the disease is seepage edema and hemorrhage around the blocking vessels. Macular cystic edema is one of the most frequently occurring complications secondary to RVO. Macular edema secondary to retinal vein occlusion (RVO-ME) leads to vision loss, even blindness, and has a great influence on people's life quality. Therefore, the patients' vision loss may be related to RVO-ME.

RVO-ME is caused by venous obstruction, which leads to damage to the retinal barrier, increasing the level of VEGF, and leakage of capillaries in the retina, resulting in fluid accumulation in the outer plexus layer of the retina and the formation of cystic edema in the macular area.

In recent years, the inflammation has been found to be related to RVO progression. Many cytokines have been identified in the vitreous cavity of patients with RVO-ME, including ICAM-1, McP-1, IL-1, IL-6, IL-8, and IL-12 [1]. However, still some controversies exist regarding RVO-ME. Several kinds of treatment are available for patients with RVO-ME, including grid laser therapy, intravitreal inject steroids, VEGF inhibitors, slow-re-releasing steroid devices, and so on [2]. At present, clear criteria for RVO-ME are lacking. Grid laser therapy is not the first treatment for RVO-ME. Anti-VEGF is the most common way to decrease macular edema. Also, drugs such as aflibercept, bevacizumab, and ranibizumab are injected into the 
vitreous humor. The safety and effect of this treatment have already been proved by many clinical trials $[3,4]$. However, the effect of anti-VEGF is maintained for at least 3 months [4], thus needing retreatment. Nowadays, anti-VEGF is given or triamcinolone actinide is injected to treat RVO-ME, leading to many complications and needing retreatment many times. Anti-VEGF needs multiple injections, leading to the requirement of patient follow-up and continuous injection and thus making patients upset. However, a few studies reported that sustained-release agents were effective to decrease intraretinal fluid and retinal hemorrhage [5]. Also, the understanding of inflammation in RVO has increased. Therefore, a large number of patients use the intravitreal injection of slow-release steroid hormones nowadays

Dexamethasone (DEX) is a synthetic, long-acting glucocorticoid with good water solubility. It produces a series of biological effects by binding to intracytoplasmic receptors and strengthens the tight connections between retinal vascular endothelial cells by inhibiting the release of inflammatory factors [5]. Ozurdex, a sustained-release intravitreal $0.7 \mathrm{mg}$ DEX delivery system, was approved for treating ME secondary to RVO. Ozurdex works for at least 4-6 months, which decreases the injection time per year, compared with anti-VEGF. DEX intravitreal implant could significantly reduce the central retinal thickness (CRT) of patients and improve the best-corrected visual acuity (BCVA) [6].

At present, no systematic review has discussed about the therapeutic effect and safety of DEX intravitreal implant in RVO-ME. Therefore, this systematic meta-analysis was performed to verify the effect and safety of BCVA and CRT in RVO-ME.

\section{Methods}

\section{Search strategy}

The study was conducted in accordance with the Cochrane Handbook for Systematic Reviews and MetaAnalysis Guidelines. The following databases were screened: PubMed, Embase-clinical key, clinicaltrials.gov, Web of science, and SinoMed. The key words were as follows: macular edema, dexamethasone intravitreal implant, dexamethasone, Ozurdex, DEX, and RVO. These words were used for search accuracy. The literature selections are shown in the PRISMA flow diagram in Figure 1.

\section{Inclusion and exclusion criteria}

The inclusion criteria were as follows: (1) patients affected by cystoid macular edema due to the RVO of recent onset (less than 3 months); (2) disease duration less than 12 weeks; (3) the DEX implant (Ozurdex) being the only intervention; and (4) comparisons of parameters before and after medication. Hence, it was decided to focus on the following primary outcomes: (1) the mean BCVA and mean improvement from the baseline in BCVA (after 3 and 6 months) obtained using the Early Treatment Diabetic Retinopathy Study; and (2) the mean CRT and mean decrease from the baseline in the CRT. The CRT was demonstrated on optical coherence tomography (after 3 and 6 months). The adverse event outcomes were as follows: (1) incidence of cataract at the end of each study; (2) elevation of intraocular pressure (IOP); and (3) mean number of Intravitreal injections. 
The exclusion criteria were as follows: diseases other than ME secondary to RVO; patients who accepted any therapies for ME secondary to RVO; studies comparing DEX with anti-VEGF; basic science or experimental study; and combined treatment.

\section{Data extraction and risk-of-bias assessment}

The relevant data were collected independently by the two authors (Xiao-Rong Gao and Yu-Jiao Dan) using a standard data extraction form. The extracted data included the publishing date, age, sex, place of the research, disease, sample size, intervention measure, injection time, outcomes, and follow-up periods. Some data of BCVA were measured in LogMAR. The following formula was used: Log MAR = $1.7-$ 0.02(letter to transform). The formula ( $S D=S E \times)$ is still used to calculate standard deviation (SD) whether the data are recorded as mean \pm standard error (SE).

Some factors were considered to show the data as mean \pm SD. RevMan 5.3 and Stata SE 15 were used to make the graphs. The Cochrane Collaboration's tool was used to assess the risk of bias about the following items: (1) sequence generation, (2) allocation concealment, (3) blinding of outcome assessor, (4) incomplete outcome data, (5) selective outcome reporting, and (6) other biases based on the Cochrane Handbook (Figure 2).

\section{Statistical analysis}

RevMan 5.3 was used to collect and analyze data. The mean difference and risk ratio were used to assess the continuous variable outcomes, and $95 \%$ confidence interval was used to assess dichotomous outcomes. The chi-square test was used to assess the heterogeneity of studies based on the values of $P$ and $P$. $P$ results between $50 \%$ and $100 \%$ represented substantial heterogeneity. $P$ values less than 0.05 were considered statistically significant.

\section{Results}

\section{Search results}

A total of 673 records from 2013 to 2018 were identified in the electronic search (PubMed, 194; Embaseclinical key, 66; Web of Science, 136; SinoMed, 145; clinicaltrials.gov, 26). After excluding 107 duplicate records, 567 studies were retained. After reading the title and abstract, 139 studies were excluded. Further, after reading the full text and considering the criteria, 30 studies were used for quantitative synthesis. Also, some studies whose final outcome was not BCVA and CRT, the results were not reported, and patients had already received other treatments were also excluded. Finally, seven eligible studies including 393 eyes were used in the present meta-analysis [7-12]. The flow chart for the study selection process is shown in Figure 1.

The characteristics of these studies are summarized in Figure 3. The sample size was from 16 to 420. Two studies were published in 2014. Three studies were published in 2016. Another study was published 
in 2017 and 2018. One study was from clinicaltrials.gov, which started in 2013 and was completed in 2015. In these studies, the same doses of DEX were given. Xiaoxi et al. reported two groups: the experimental group received $0.7 \mathrm{mg}$ DEX injection, and the control group was given the sham injection (pressed against a needleless applicator on the conjunctiva). Daniele et al performed the follow-up 3 months after the injection. Simone et al performed follow-up evaluations after 1, 3, and 6 months. Yoon et al followed up after 6 and 12 months. The risk-of-bias assessment results are presented in Figure 2 .

\section{Meta-analysis results}

\section{Mean BCVA after 3 months}

Two studies including 54 eyes reported the BCVA at 3 months. No difference in treatment effect on BCVA was found 3 months before and after the medication. The MD in patients' vision after injecting DEX was 10.86 ( $95 \% \mathrm{Cl}:-0.13$ to $21.85, P=0.05$, Figure 4 ). Also, no statistical heterogeneity was reported (chisquared: $0.01, P=0.94$, tsquared: $0 \%)$.

\section{Mean BCVA after 6 months}

Six studies including about 171 eyes reported that BCVA after 6 months improved from the baseline, with obvious differences after injecting DEX. The MD in patients' vision after injecting DEX was 15.66 (95\% Cl: 9.68-21.65, $P=0.00001$, Figure 5). Also, statistical heterogeneity was reported (chi-squared: $4.68, P=$ 0.32 , 1 -squared: $14 \%)$.

\section{Mean CRT after 3 months}

Two studies of 54 eyes reported data on CRT 3 months after the initial treatment. Both involved a reduction of CRT from the baseline. The MD for the two studies 3 months after injection was significant $(\mathrm{MD}=-199.20,95 \% \mathrm{Cl}:-408.19$ to $9.79, P=0.06)$. It showed that injecting DEX was associated with no statistical heterogeneity; high heterogeneity was found in the first 3 months $(P<0.0001,1$ squared $=94 \%$; Figure 6).

\section{Mean CRT after 6 months}

Six studies of 171 eyes reported data on CRT after 6 months and showed a significantly large reduction from the baseline. The MD of these studies showed an obvious change (MD $=-207.56,95 \% \mathrm{Cl}:-275.03$ to $-140.10, P=0.00001$, Figure 7$)$. Also, high heterogeneity was noted $(P=0.002$, 1 squared $=74.9 \%$; Figure 7).

\section{Elevation of IOP}

Some patients demonstrated an increase in IOP after injecting DEX. This might have been caused by the surgery or some postoperative reaction. No heterogeneity was noted $(P=0.54$, - -squared $=0 \%$; Figure 7$)$. It explained why the IOP of patients after injecting DEX showed a significant difference $(\mathrm{RR}=29.33 ; 95 \% \mathrm{Cl}$ : 9.38-91.76; $P=0.00001$ ). (Figure 8) 
Two studies involving 71 eyes demonstrated 17 eyes with postoperative cataract. A significant difference was found after injecting DEX (RR $=18,95 \% \mathrm{Cl}: 2.47-130.99, P=0.004)$ and without heterogeneity $(P=$ 0.78, - squared $=0 \%$; Figure 9 ).

\section{Discussion}

In this meta-analysis, seven studies were selected to evaluate the safety and efficacies of DEX implants in treating RVO. DEX could improve visual function and changes in fundus structure during treatment. No difference in the treatment effects on BCVA or CRT 3 months before and after medication and no statistical heterogeneity were found. However, a significant improvement in BCVA was observed 6 months before injecting DEX, with statistical significance. At the same time, the analysis demonstrated that DEX implant could obviously improve BCVA and decrease the CRT after 6 months. However, in the first 3 months, no significant improvement and reduction in BCVA and CRT were observed. This might be related to the slow release of DEX. At first, the concentration of drugs in the vitreous cavity was relatively low. With the extension of time, the drug was released gradually, and the drug concentration reached the peak. At this moment, the medicinal effect was the best. The drug concentration then stabilized as the metabolism in the body gradually decreased [3].

At present, an intravitreal injection of anti-VEGF is considered as the preferred treatment for RVO. The pathogenesis of RVO is still unclear. Some scientists believe that retinal vein blood flow does not cause vascular obstruction, blood stasis in blood vessels, and increase in capillary pressure, resulting in retinal bleeding, exudation, reduction in capillary perfusion, and retinal ischemia caused by the release of a large number of vascular endothelial factors. Hence, it is presumed that macular edema secondary to RVO can be decreased by resisting the release of vascular endothelial factors. Anti-VEGF becomes the main treatment in the short term. However, it has some limitations: monthly injections are required, causing inconvenience; repeated injections lead to some difficulties, for example, wastage of time in treatment, increased risk of infection, intraocular inflammation, and so on[13]; and the treatment is also expensive for most patients. Therefore, another study found that RVO was also related to inflammatory cytokines causing inflammatory diseases, including chemokines, prostaglandins, matrix metalloproteinase, interleukins, selectins, vascular cell adhesion molecule-1 (VCAM-1), intercellular adhesion molecule-1 (ICAM-1), angiotensin II, and inflammatory cells (macrophages and neutrophils) [14]. It is believed that RVO can be effectively relieved by controlling the release of inflammatory factors. An intravitreal injection of DEX is an effective way because the drug can be released slowly and continuously in the vitreous cavity for at most 6 months [14]. Glucocorticoid can effectively control inflammation and edema. DEX is useful in treating RVO, decreasing the time of injection and the chance of infection. Thus, DEX implant may be a better way for treating RVO compared with other treatments.

According to some studies, the outcomes, such as BCVA and center retinal thickness, are often considered as important measures of treatment efficacy. The outcome measures in the present study 
were the same as in other studies.

At present, an intravitreal injection of DEX preparation is increasingly accepted by the majority of patients. The implantation of sustained-release devices in the vitreous cavity is a novel treatment method introduced in clinical practice. Several basic experimental studies have proved that DEX has a good alleviating effect on macular edema secondary to RVO. At present, a number of studies have shown that this disease has an inflammatory response with inflammatory factors. DEX is a corticosteroid hormone that can effectively inhibit inflammatory factors and maintain the pharmaceutical concentration in the vitreous cavity with a long half-life, which solves the inconvenience of patients with repeated injections in the past. At the same time, the drug implant is rod-shaped, about $6 \mathrm{~mm}$ long and less than $0.5 \mathrm{~mm}$ in diameter, and can be completely degraded without removal. The injection instrument uses $22 \mathrm{G}$ precision needles and innovative drug thrusters, which makes the injection easier, effectively reduces the difficulty of surgery, and facilitates surgeons. Therefore, DEX is the most popular ophthalmic treatment at present and is gradually accepted by patients and ophthalmologists.

The cost and convenience also need to be considered when applying therapies. DEX can maintain its effective dose in the vitreous cavity for about 6 months [15], which decreases the time of injection compared with anti-VEGF and reduces the risk of infection. One study analyzed the cost of these therapies. Multiple injections cost a lot of money, and many patients finally chose to give up because they could not afford the high cost. The average number of DEX injections was reduced. Although the cost of a single injection was higher, the long-term cumulative cost was significantly lower than that of anti-VEGF [16].

However, an intravitreal injection of DEX inevitably leads to some common complications. In this metaanalysis, the data demonstrated that SAEs, such as intraocular hypertension and postoperative cataract, could easily happen in the DEX implant, showing statistically significant differences between before and after medication [17]. Some studies reported that previous pars plana vitrectomy, an open/defective lens capsule, and/or iris defects may increase the risk of DEX implant migration into the anterior chamber [15]. Therefore, for patients with a history of vitrectomy, anterior chamber surgery, and lens capsule damage, extreme care is needed when injecting DEX into the vitreous cavity.

Due to the limited number of included studies and other limitations, subgroup analysis on the heterogeneity of studies to find the source of heterogeneity could not be conducted. It was presumed that the heterogeneity of 3-month CRT mainly derived from the small sample size, age of participants, and a higher proportion of females. Further, the CRT was disturbed by many factors, such as individual difference, measurement error, individual sensitivity to medication, and actual dosage of medication administered. The heterogeneity of mean change in the CRT after 12 months was caused by the studies of Chun- Ju Lin et al [8] and Mariacristina et al [9]. In these two studies, some patients were injected more than one time. It increased the doses of DEX, affecting the result of CRT. Many factors, such as age, baseline data, race, dosage, and way of injecting, might affect the heterogeneity. However, some factors 
could not be removed. Similar to the results of relevant studies, the present study showed a significant decrease in the CRT in patients after DEX injection in the vitreous body for 6 months.

This meta-analysis had some limitations: (1) it included only six studies, and hence the total sample was relatively small. (2) Some studies proceeded for only 3 months, and hence the incidence of postoperative adverse reactions could not be accurately assessed. (3) At the same time, due to the different age range of the participants, it was not possible to accurately assess the preoperative vision and fundus condition of the patients. At the same time, different drug sensitivity of the patients led to greater heterogeneity. (4) The follow-up was performed for only 6 months and hence the efficacy of DEX at a later stage could not be evaluated accurately.

\section{Conclusions}

In summary, the meta-analysis of six clinical trials showed that intravitreal DEX could effectively reduce macular edema in patients with RVO, especially after 3-6 months of medication. The patients' bestcorrected vision acuity also significantly improved after 3-6 months. The effect of the drug lasted for about 6 months, reducing the patients' trouble with multiple injections. However, the use of DEX was also associated with some problems, such as postoperative high intraocular pressure and increased risk of cataract, which requires further improvement in future studies to reduce postoperative adverse reactions.

\section{List Of Abbreviations}

BCVA, best-corrected visual acuity

CRT, central retinal thickness

DEX, dexamethasone

ICAM-1, intercellular adhesion molecule-1

IOP, intraocular pressure

RVO, retinal vein occlusion

RVO-ME, macular edema secondary to retinal vein occlusion

SD, standard deviation

SE, standard error

VCAM-1, vascular cell adhesion molecule-1

\section{Declarations}




\section{Ethics approval and consent to participate}

All analyses were based on previous published studies, thus no ethical approval and patient consent are required.

\section{Consent for publication}

Not applicable.

\section{Availability of data and material}

The datasets used and/or analysed during the current study are available from the corresponding author on reasonable request.

\section{Competing interests}

The authors declare that they have no conflict of interest.

\section{Funding}

This work was supported by the Youth Innovation in Medical Research in Sichuan Province(Q15014), LuZhou Municipal People's Government-Sichuan Medical University Science and Technology Strategic Cooperation Project(2015LZCYD-S06(1/11)), Scientific Research Topics of Sichuan Health and Family Planning Commission(16PJ560), Affiliated Hospital of Luzhou Medical College scientific research project(2015-PT-017)

\section{Authors' contributions}

Xiaorong Gao and Yue He conceived and coordinated the study, designed, performed and analyzed the experiments, wrote the paper. Jie Chen, Yujiao Dan and Gang Tian carried out the data collection, data analysis, and revised the paper. All authors reviewed the results and approved the final version of the manuscript.

\section{Acknowledgements}

Not applicable

\section{References}

1. Ascaso FJ, Huerva V, Grzybowski A. The role of inflammation in the pathogenesis of macular edema secondary to retinal vascular diseases. Mediators Inflamm. 2014;2014:432685.

2. Jonas JB. Medical Therapy for Macular Edema Secondary to Retinal Vein Occlusion. Asia Pac J Ophthalmol (Phila). 2016;5:93-4. 
3. Chang-Lin JE, Attar M, Acheampong AA, Robinson MR, Whitcup SM, Kuppermann BD, et al. Pharmacokinetics and pharmacodynamics of a sustained-release dexamethasone intravitreal implant. Invest Ophthalmol Vis Sci. 2011;52:80-6.

4. Li X, Wang N, Liang X, Xu G, Li XY, Jiao J, et al. Safety and efficacy of dexamethasone intravitreal implant for treatment of macular edema secondary to retinal vein occlusion in Chinese patients: randomized, sham-controlled, multicenter study. Graefes Arch Clin Exp Ophthalmol. 2018;256:59-69.

5. FENG Y-B, ZHU J-Y, WENG W-Q. Research progress On biodegrad able hormone sus-tained release agent Ozurdex for macular edema. 2016.

6. DANIS RP, SADDA S, JIAO J, LI X-Y, WHITCUP SM. The Relationship Between The Retina Eye Thickness And Vision Retinal Vein Occlusion Treat With Dexamethasone The Implants. 2016.

7. Donati S, Gandolfi C, Caprani SM, Cattaneo J, Premoli L, Azzolini C. Evaluation of the Effectiveness of Treatment with Dexamethasone Intravitreal Implant in Cystoid Macular Edema Secondary to Retinal Vein Occlusion. Biomed Res Int. 2018;2018:3095961.

8. Lin CJ, Chen HS, Su CW, Tien PT, Lin JM, Chen WL, et al. The Effect of Age and Initial Central Retinal Thickness on Earlier Need of Repeat Ozurdex Treatment for Macular Edema Due to Retinal Vein Occlusion: A Retrospective Case Series. J Ocul Pharmacol Ther. 2017;33:763-72.

9. Parravano M, Oddone F, Boccassini B, Giorno P, Chiaravalloti A, Tedeschi M, et al. Exploring the morphological and functional retinal changes after dexamethasone intravitreal implant (Ozurdex(R)) in macular edema due to retinal vein occlusion. Ophthalmic Res. 2014;51:153-60.

10. Veritti D, Macor S, Lanzetta P. Early effects of dexamethasone implant on macular morphology and visual function in patients with macular edema secondary to retinal vein occlusion. Ophthalmologica. 2014;232:144-8.

11. Yoon YH, Kim JW, Lee JY, Kim IT, Kang SW, Yu HG, et al. Dexamethasone Intravitreal Implant for Early Treatment and Retreatment of Macular Edema Related to Branch Retinal Vein Occlusion: The Multicenter COBALT Study. Ophthalmologica. 2018;240:81-9.

12. Michalska-Małecka K, Gaborek A, Nowak M, Halat T, Pawłowska M, Śpiewak D. Evaluation of the effectiveness and safety of glucocorticoids intravitreal implant therapy in macular edema due to retinal vein occlusion. 2016 .

13. Arikan Yorgun M, Mutlu M, Toklu Y, Cakmak HB, Cagil N. Suspected bacterial endophthalmitis following sustained-release dexamethasone intravitreal implant: a case report. Korean $\mathrm{J}$ Ophthalmol. 2014;28:275-7.

14. Chang-Lin JE, Burke JA, Peng Q, Lin T, Orilla WC, Ghosn CR, et al. Pharmacokinetics of a sustainedrelease dexamethasone intravitreal implant in vitrectomized and nonvitrectomized eyes. Invest Ophthalmol Vis Sci. 2011;52:4605-9.

15. Rahimy E, Khurana RN. Anterior segment migration of dexamethasone implant: risk factors, complications, and management. Curr Opin Ophthalmol. 2017;28:246-51.

16. Smiddy WE. Economic considerations of macular edema therapies. Ophthalmology. 2011;118:182733. 
17. Garweg JG, Zandi S. Retinal vein occlusion and the use of a dexamethasone intravitreal implant (Ozurdex(R)) in its treatment. Graefes Arch Clin Exp Ophthalmol. 2016;254:1257-65.

\section{Figures}

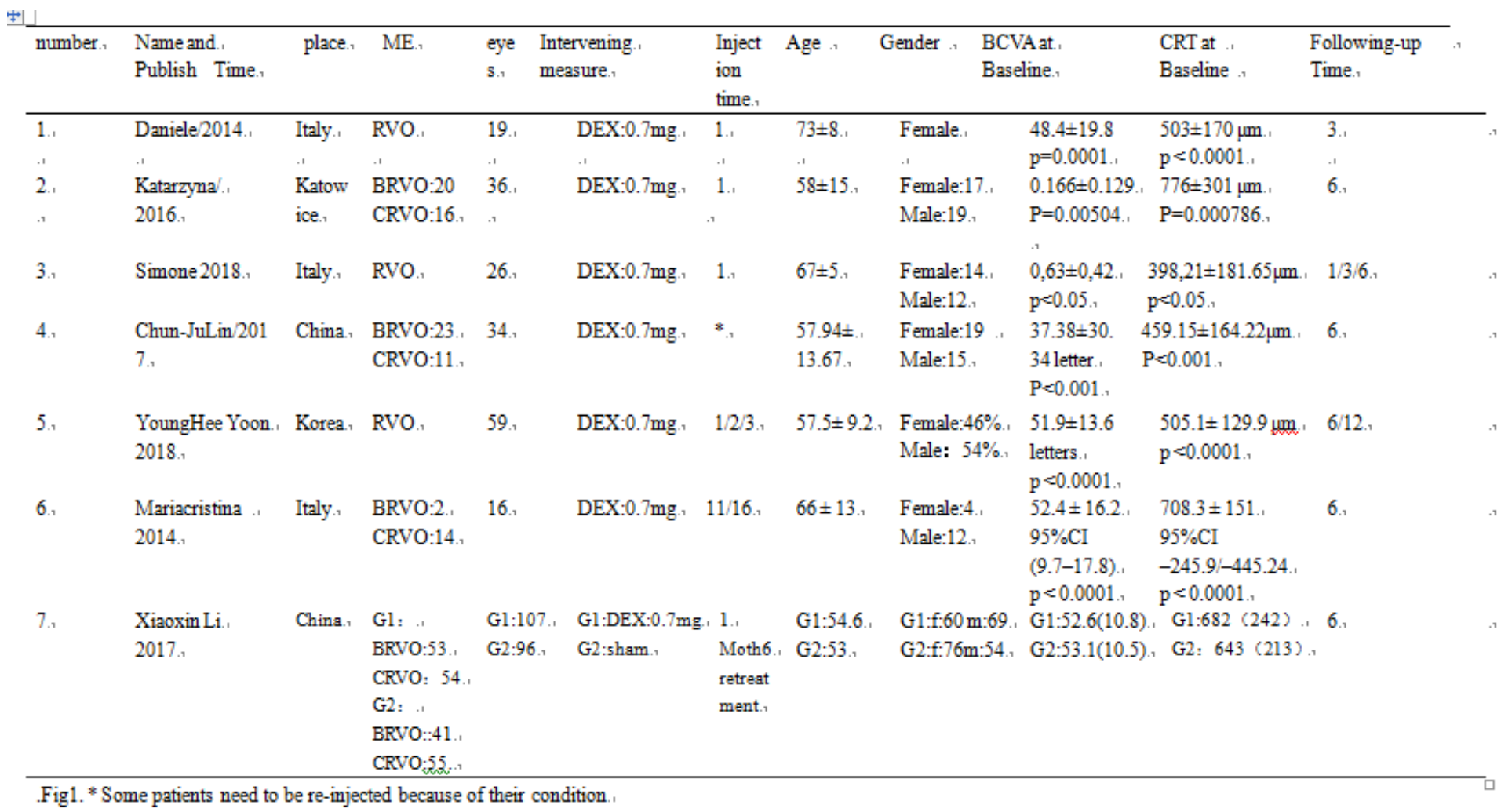

\section{Figure 1}

Flow chart for study search. 


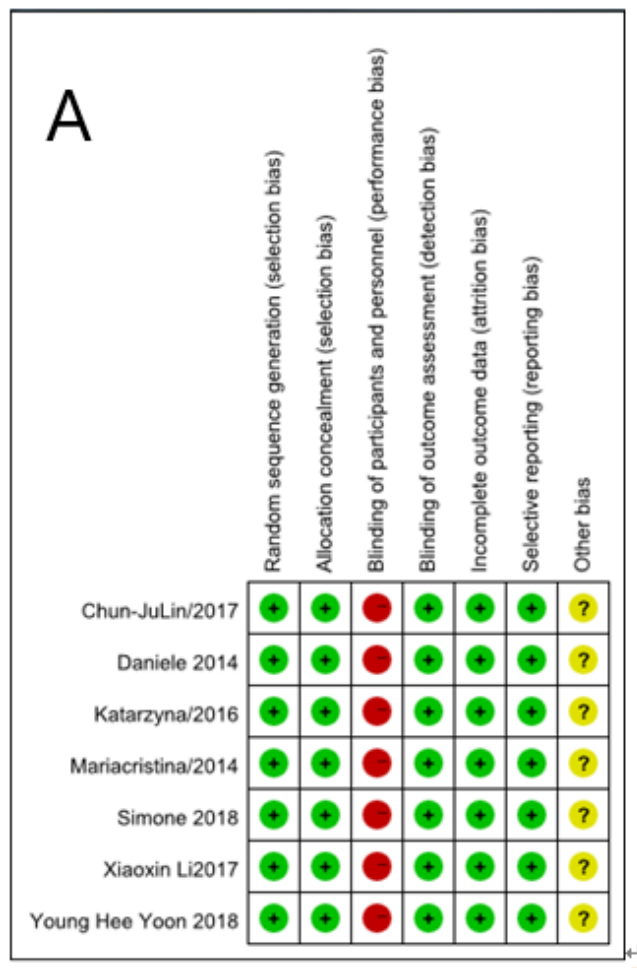

B

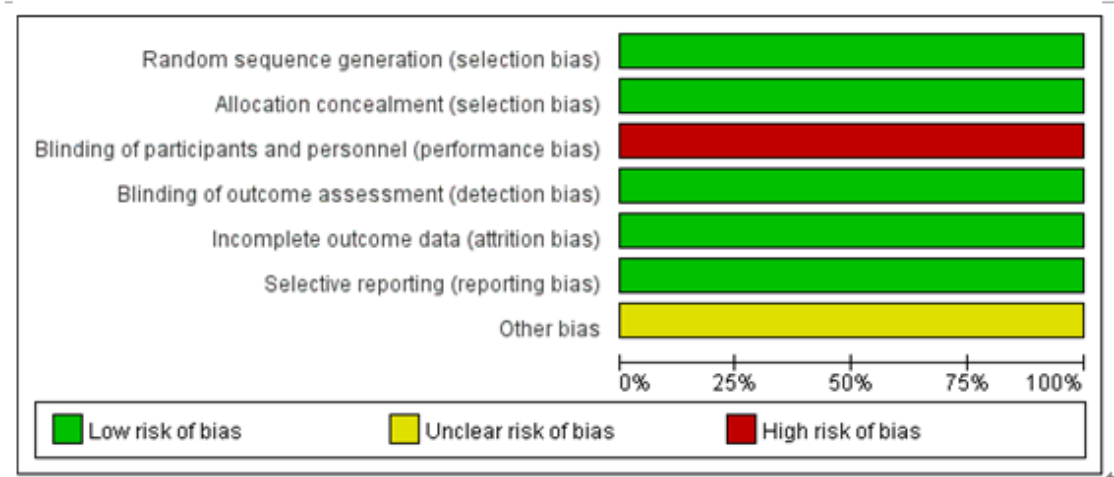

\section{Figure 2}

Evaluated risk of included studies. (A) Assessment of risk of bias: +, low risk of bias; -, high risk of bias;?, unclear risk of bias. (B) Risk-of-bias graph: Risk assessment for included studies, expressed as a percentage. 


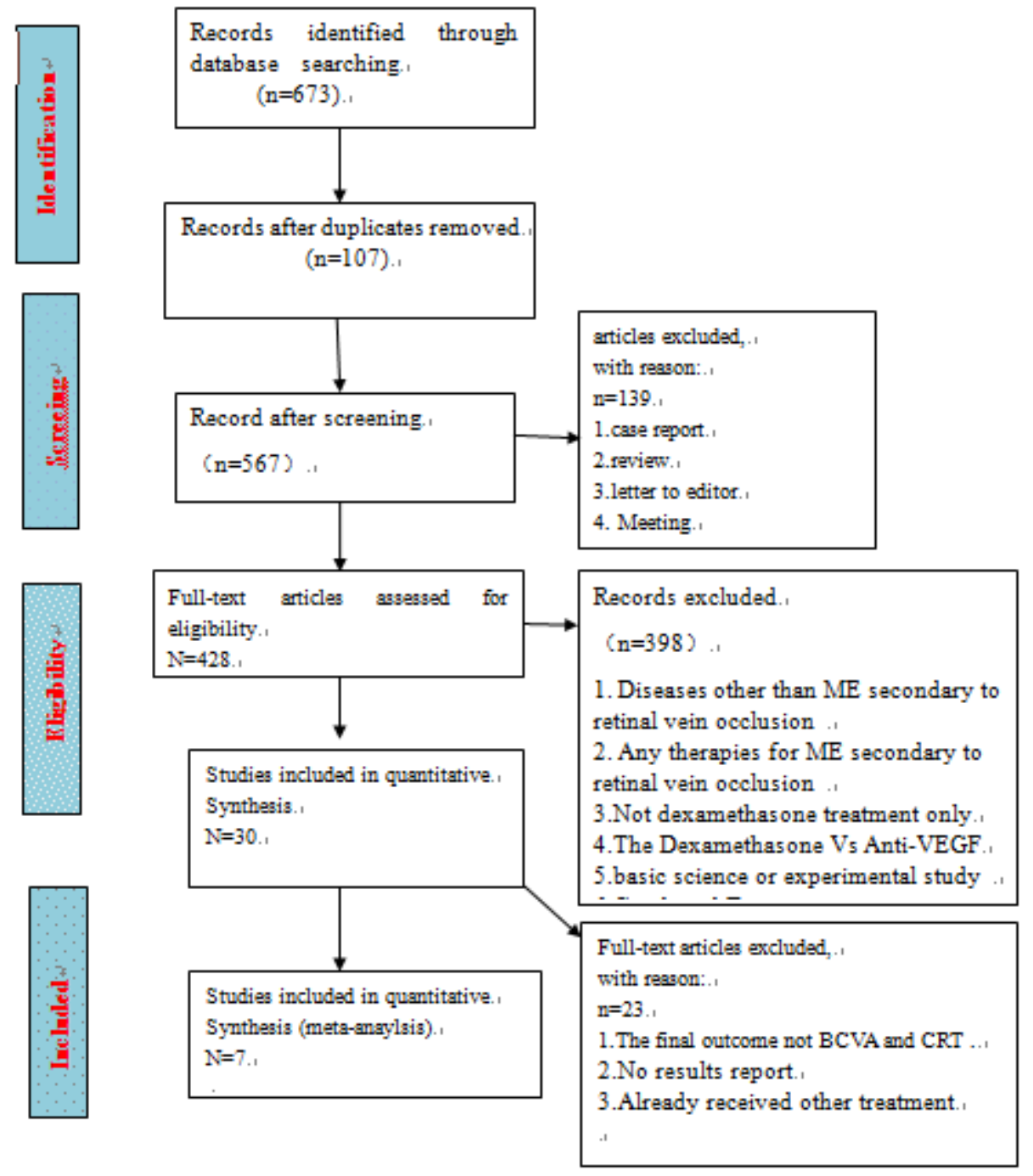

\section{Figure 3}

Summary of the Characteristics of the included studies

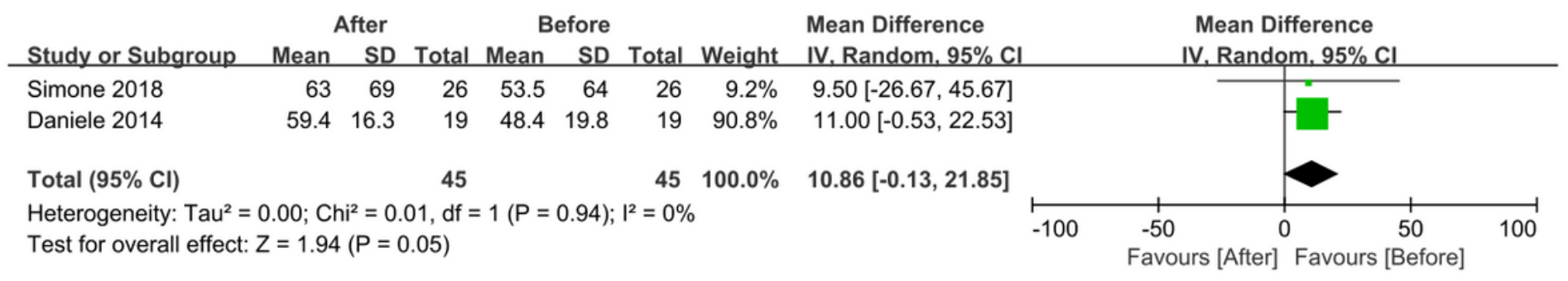

\section{Figure 4}

Forest plot showing the change in average BCVA and $95 \% \mathrm{Cl}$ after 3 months of medication. 


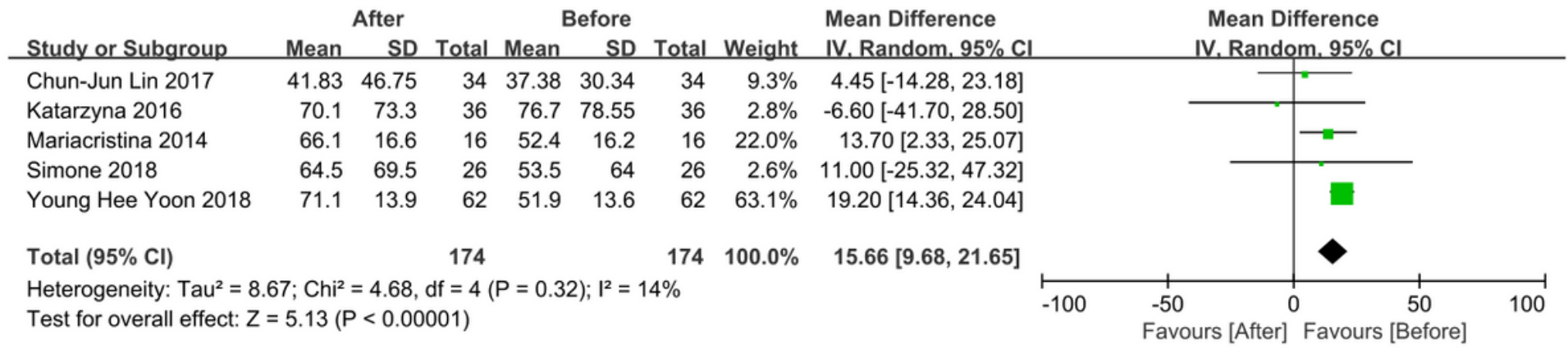

\section{Figure 5}

Forest plot showing the change in average BCVA and $95 \% \mathrm{Cl}$ after 6 months of medication.

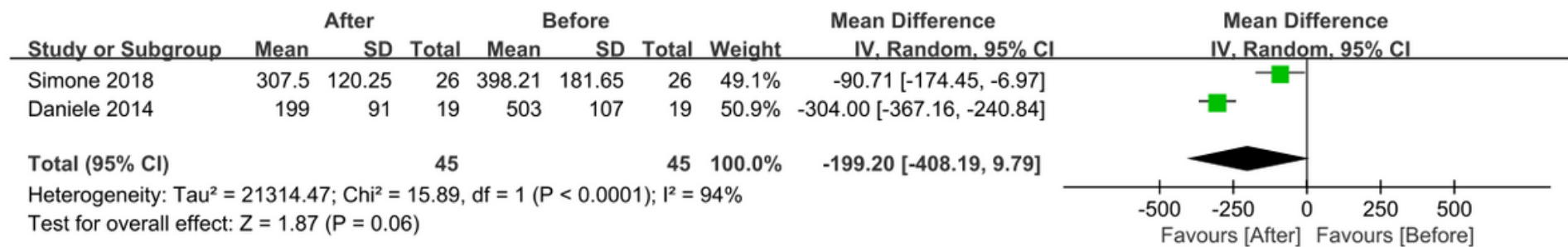

\section{Figure 6}

Forest plot shows the change in the average CRT and $95 \% \mathrm{Cl}$ after 3 months of medication.

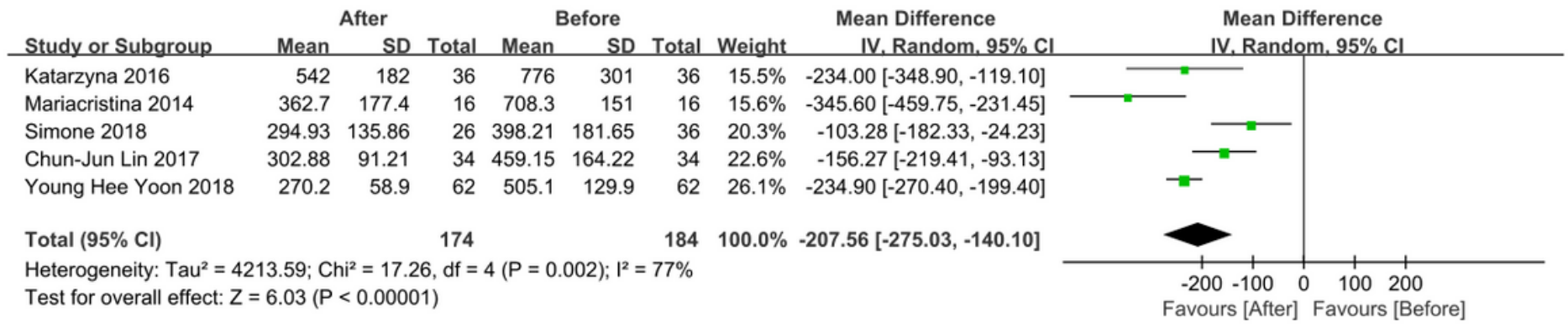

\section{Figure 7}

Forest plot showing the change in the average CRT and 95\% Cl after 6 months of medication. 


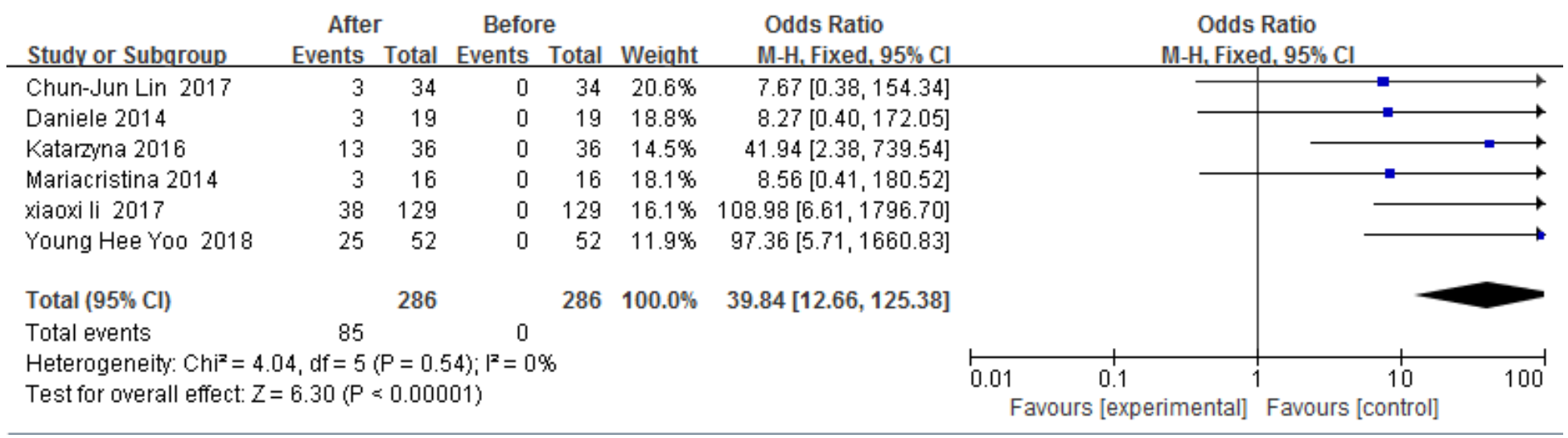

\section{Figure 8}

Forest diagram showing the changes in IOP after administration.

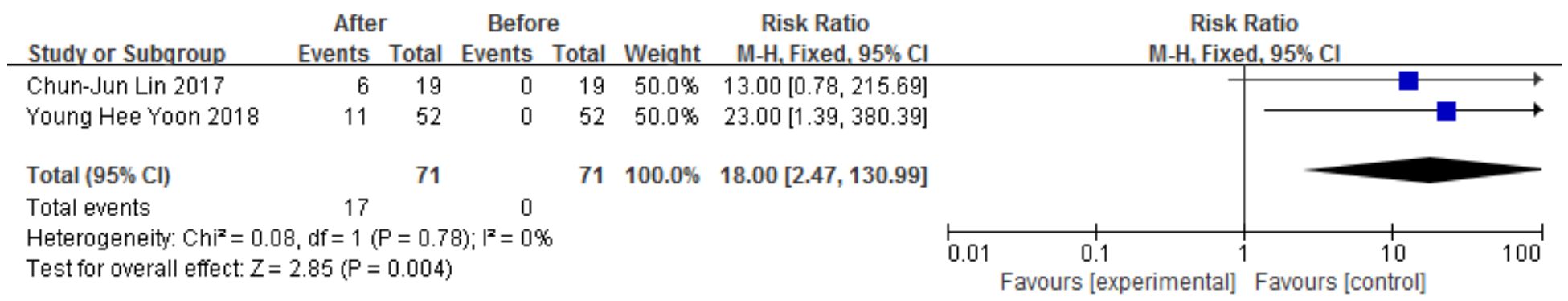

\section{Figure 9}

Forest diagram showing the changes in cataract after administration. 